\title{
The Use of Handheld Ultrasound Devices in Emergency Medicine
}

\author{
Adrienne N. Malik ${ }^{1} \cdot$ Jonathan Rowland ${ }^{2} \cdot$ Brian D. Haber $^{3} \cdot$ Stephanie Thom ${ }^{1} \cdot$ Bradley Jackson $^{1}$. \\ Bryce Volk ${ }^{1} \cdot$ Robert R. Ehrman ${ }^{3}$ (D)
}

Accepted: 21 April 2021 / Published online: 11 May 2021

(C) The Author(s), under exclusive licence to Springer Science+Business Media, LLC, part of Springer Nature 2021, corrected publication 2021

\begin{abstract}
Purpose of Review Ultraportable handheld ultrasound (HHU) devices are being rapidly adopted by emergency medicine (EM) physicians. Though knowledge of the breadth of their utility and functionality is still limited compared to cart-based systems, these machines are becoming more common due to ease-of-use, extreme affordability, and improving technology.

Recent Findings Images obtained with HHU are comparable to those obtained with traditional machines but create unique issues regarding billing and data management. HHU devices are increasingly used successfully to augment the education of practitioners-in-training, by emergency physicians in austere environments, and in the burgeoning fields of "tele-ultrasound" and augmented reality scanning.

Summary This review seeks to describe the current state of use of HHU devices in the emergency department (ED) including device overview, institutional concerns, unique areas of use, recent literature since their adoption into clinical EM, and their future potential.
\end{abstract}

Keywords Handheld ultrasound · Emergency ultrasound · POCUS · Pocket ultrasound · Portable ultrasound · Emergency medicine

\section{Introduction}

Point-of-care ultrasound (POCUS) is ubiquitous in emergency departments (EDs) and recognized as standard of care in the workup of multiple disease processes encountered by the emergency physician (EP). In addition to its recognition as a workhorse for diagnosis and procedural guidance, POCUS is increasingly used by other medical specialties, ancillary staff, and first responders. POCUS is increasingly used outside the clinical setting as a tool for teaching practitioners-in-training

This article is part of the Topical collection on Technology in Medicine

Robert R. Ehrman

rehrman@med.wayne.edu

1 Department of Emergency Medicine, University of Kansas School of Medicine, 3901 Rainbow Blvd, Kansas City, KS 66045, USA

2 Department of Emergency Medicine, University of California-Irvine, 1001 Health Sciences Rd, Irvine, CA 92617, USA

3 Department of Emergency Medicine, Wayne State University School of Medicine, 4201 St. Antoine, Suite 6G, Detroit, MI, USA with ultrasound instruction integrated into medical school curricula.

Technological advances have led to the introduction of truly handheld ultrasound (HHU) into the POCUS market [1•]. With smaller footprints, user friendly interfaces, and lower price points, the broad appeal of these devices is clear. However, as with most emerging technology in healthcare, the advent of HHU devices brings a host of clinical and academic potential as well as new challenges and complex questions about the handling of patient data.

While numerous HHU devices are available, and large, robust data to support their interchangeable use with the traditional machines is lacking $[2,3 \cdot \bullet]$, the American College of Emergency Physicians (ACEP) released a consensus statement recognizing HHU-generated images as "comparable" to those of traditional machines $[1 \cdot, 2-4]$. In order to better navigate the myriad handheld devices, this paper will serve as an overview of what is currently known about HHU devices: the mechanics of how they work, nuances of their use in practice, how they compare to traditional machines, and what this technology portends for the future of POCUS in the ED and beyond. 


\section{Devices}

Several HHU devices are currently available. A comparison of specifications between several devices can be found in Fig. 1. The image-generating technology between handhelds and traditional machines is similar with one exception. To create ultrasound images, traditional cart-based ultrasound systems and most handhelds use piezoelectric crystal technology. The product from Butterfly Network Inc., however, utilizes capacitive micromachined ultrasound transducers on complementary metal oxide semiconductors, or CMUT-on-CMOS technology. CMUTs replace the traditional vibrating piezoelectric crystals with oscillating drums embedded in a single silicon chip, serving the same function of converting electricity into sound waves [4, 5]. In addition to decreasing the cost of production, this technology allows a single device to operate at a wider range of frequencies, negating the need for multiple probes [5].

Handheld products come as single or multiple probe devices. SonoQue and GE offer two separate heads on a single probe, increasing the range of studies that can be performed by simply rotating the probe (Fig. 1) [3・•]. While some products have the ability to display generated images on personal smart devices, others utilize proprietary displays sold with the product, which may hold much of the processing power of the system [3••]. Connectivity between the probe and the display varies between products. Some HHU probes connect through a traditional cable system, while some products offer wireless communication. The Clarius and Vave devices can stream their images live to multiple displays simultaneously.

Some HHU devices feature artificial intelligence (AI) packages that perform calculations such as ejection fraction and bladder volume measurement [5]. Image recognition software allows auto-identification and labeling of structures and gives real-time feedback on the quality of the images obtained. The Kosmos product can also perform digital cardiac auscultation with image acquisition and comes with telemetry leads which can be attached to the patient, thus integrating electrocardiograph findings and rhythm interpretation into the ultrasound study.

Understanding the benefits and limitations of HHU is critical to its proper use in the clinical arena. In the same way a POCUS examination is not a comprehensive evaluation, HHU excels when used to evaluate well-defined clinical questions

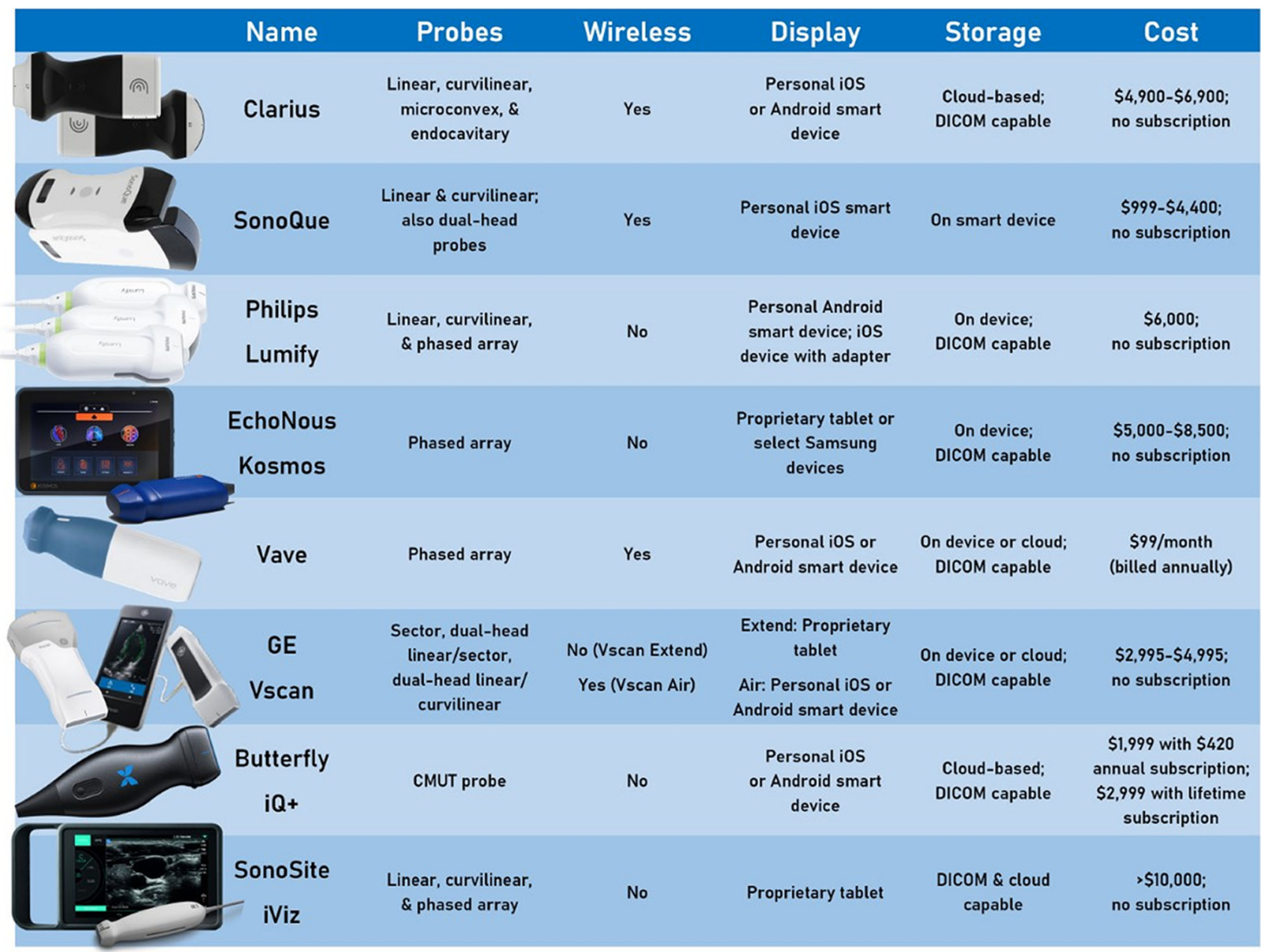

Fig. 1 Comparison of specifications between several devices 


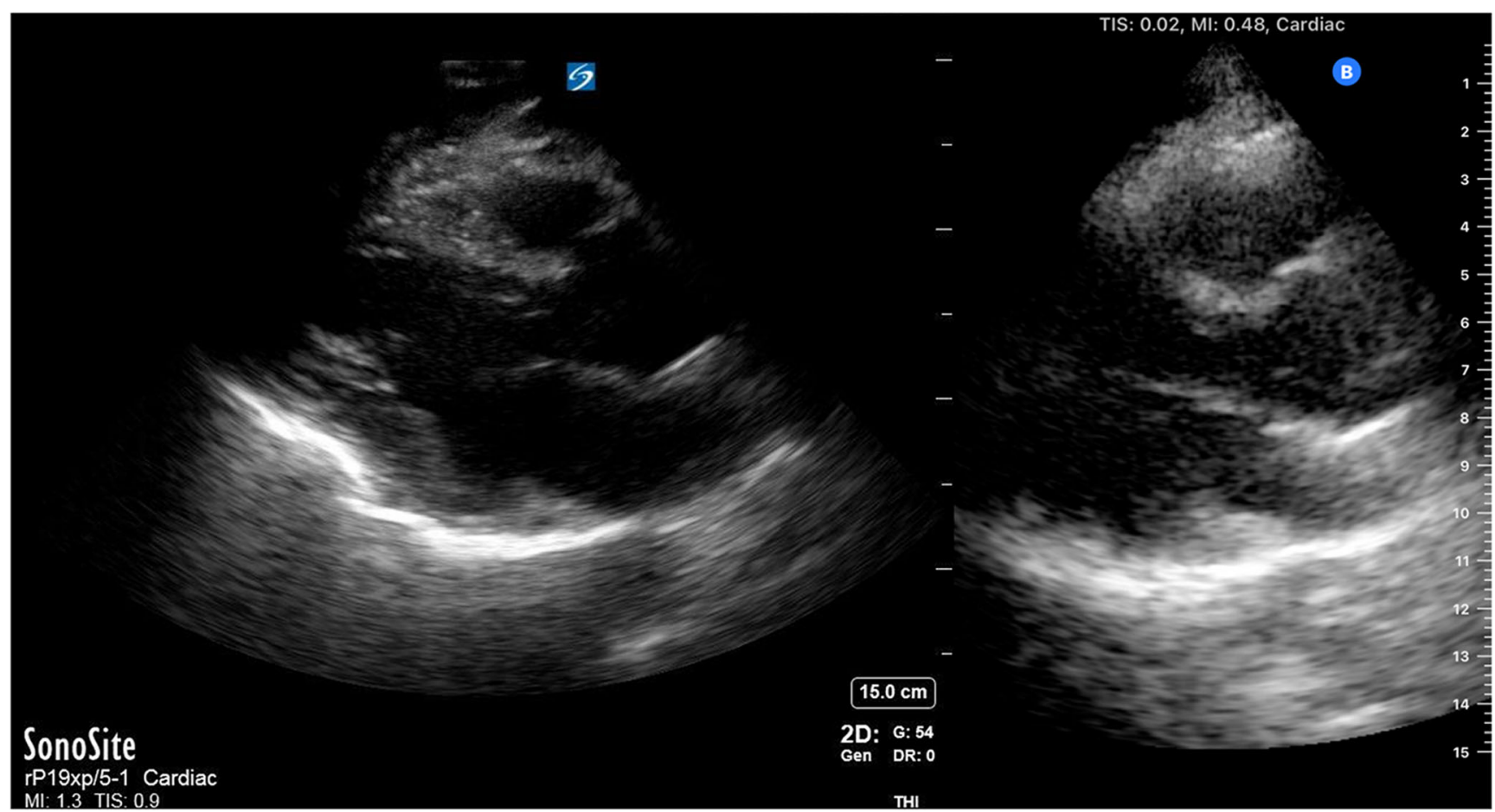

Fig. 2 Side by side comparison of a parasternal long axis (PLAX) cardiac view obtained on the same patient using a SonoSite X-Porte (Fujifilm, SonoSite INC. Bothell, WA) on the left and the Butterfly iQ HHU

in a manner that respects the limitations of this new technology $[3 \cdot \bullet, 5]$.

A primary benefit to $\mathrm{HHU}$ is that it is readily available to the clinician for every patient encounter. Starting as low as $\$ 2000$, these devices are the cheapest available ultrasound (US) systems, opening the door for widespread use in medical education, resource-limited settings, or to any clinician who performs POCUS examinations $[2,3 \cdot \bullet, 6,7]$.

Most HHU devices offer a battery life that is capable of intermittent scanning for the duration of a clinical shift without being tethered to an electrical outlet $[5,8,9]$. However, this benefit is tempered by the tendency of some devices to overheat after a relatively short period of use $[5,8]$. Image quality of $\mathrm{HHU}$ has a foundation of published literature establishing its non-inferiority to cart-based machines for POCUS applications ${ }^{1}$, though anecdotally HHU-generated images are subjectively of lesser quality (Fig. 2) $[2,4,5,8-10 \bullet \cdot$. A notable exception is that CMUT-onCMOS (i.e., Butterfly iQ) technology has minimal published comparisons against traditional machines. Advanced echocardiographic applications are also lacking in the HHU world. Focused cardiac US can still be qualitatively performed with HHU, but few current devices offer spectral Doppler capabilities, thus precluding their use for quantitative applications $[5,8,11,12 \cdot 13]$.

\footnotetext{
${ }^{1}$ Of note, two systematic reviews on handheld ultrasound diagnostic performance are heavily dominated by studies that utilized GE's Vscan device. Ninety-four percent of studies included by Rykkje et al. [3.•] and $88 \%$ by Galusko et al. $[10 \bullet \cdot]$ were on the Philips Healthcare Vscan.
}

(Butterfly Network INC, Guilford, CT) on the right. Both images were obtained using the cardiac preset function on each machine

Most HHU models leverage app-based smartphone technology, generally Android or iOS [2] making boot times negligible and reducing complicated knobology to familiar finger swipes and taps [14], thereby reducing the barrier to entry for the novice users. Many HHU models offer HIPAA-compliant cloud-based storage with a user interface that promotes collaboration and immediate feedback from remote reviewers without the need for a middleware solution $[3 \bullet \bullet, 5,15]$. Cloud-based storage is often proprietary and may be incapable of agnostic integration with existing US and/or hospital Picture Archiving and Communication System (PACS) systems, but most HHU systems can be configured for DICOM PACS [2]. HIPAA compliance is also contingent upon the device/smartphone with which the HHU is paired. The use of powerful smart devices opens opportunities for teleultrasound and remote guidance directly from the device itself without the need for additional equipment [16].

\section{Institutional Concerns}

\section{Image Storing and Sharing}

Archiving images and clips for all POCUS exams is best practice and required for billing. HHU devices offer novel solutions to image archiving $[5,8]$. When deciding between local PACS vs. cloud-based storage, most of the handheld devices 
allow either option [2]. Choosing the local storage option most closely mirrors existing workflows with cart-based machines and likely allows more seamless integration with existing PACS systems. Cloud-based image archiving on the other hand prompts important questions about ownership of images, HIPAA compliance, and security of images.

While image ownership is a complex legal question, the precedent is that it is owned by the creator of the information, i.e., the clinician. Butterfly IQ follows this precedent in their Patient Privacy Notice, identifying themselves as "data processor" and clinicians as "data controller" [17, 18]. This same terminology is used by another major cloud storage solution, Trice Imaging [19]. The term "data controller" originates from the European Union's General Data Protection Regulation and indicates a party who decides what to do with data and when to delete it. Physician ownership of imaging studies is further substantiated in that patient requests to modify or remove data sent to Butterfly will be referred back to the clinician who obtained the image [20]. Likewise, Trice Imaging provides tools for clinicians to modify or delete studies but will not do so themselves [19]. Another important ethical consideration with image ownership is that some cloud-based services use de-identified ultrasound studies from users to improve proprietary AI algorithms, which will then be sold back to the same users.

A hospital-owned PACS server is the traditional workflow solution for image archiving, but significant and common security issues have been identified [2, 21, 22]. Cloud-based storage brings its own set of security concerns, in part due to the increased visibility [17]. Despite these concerns, cloud storage offers security advantages over local storage as IT resources are pooled, making it easier to stay up-to-date with threats [23]. Security of ultrasound images is an important and complex topic which requires further investigation.

Other factors that ultimately influence a hospital's decision between local or cloud storage include internet connectivity, bandwidth availability, and redundancy of image availability during "downtime." All of these factors require careful consideration at the hospital level and are best undertaken prior to implementation of the new technology $[1 \bullet]$.

\section{Billing}

Per ACEP guidelines, "ultrasound is a separate entity from the physical examination that adds anatomic, functional and physiologic information to the care of the acutely-ill patient" [24]. Comparisons to stethoscopes are inaccurate, as ultrasound presents a new set of data separate from the physical examination that requires archiving and quality assurance [25]. Similar to other procedures, appropriate performance and interpretation of ultrasound requires training, skill development, feedback, credentialing, and time to complete the study. Therefore, imaging studies completed with handheld devices should continue to be billed as independent procedures and not regarded as an extended physical examination $[1,24]$.

Ownership of the device impacts the type of billing allowed. Hospital device ownership is preferable, allowing billing for both facility and professional fees [2]. It is unlikely that the facility fee can be billed for devices owned by individuals or physician groups. Facility fees are needed to support overhead costs of machine purchases, EMR integration, maintenance, and workflow solutions. This also has the potential to create confusion for coding and billing companies, as device ownership may not be readily apparent [2]. Without approval from hospital administration, IT, security, and legal departments, ACEP's stance is that personally owned devices should not be used clinically [1•]. Overall, it is recommended that the hospital purchase both the HHU transducer and the device with which it will be used - this will allow for both the professional and technical fees to be charged without changes to extant billing structures [2].

\section{Theft/Misplacement}

The portability offered by handheld devices is a double-edged sword, as they are more prone to misplacement or theft [5]. Though data is ultimately uploaded, some protected health information (PHI) is maintained on the portable device. To safeguard health information and comply with HIPAA, devices should ideally be hospital-owned and passwordprotected. They should also be loaded with mobile device management software allowing tracking, locking, and erasure of PHI. If enough devices can be purchased, assigning devices to individual providers may allow greater personal responsibility over their safekeeping. Radio frequency identification tagging is another option commonly implemented in hospitals to track device location and provide alerts if a device leaves the premises.

\section{Disinfecting Procedures and Concerns}

Proper cleaning of HHU devices is necessary to prevent iatrogenic infection transmission. Cleaning processes for handheld devices are similar to cart-based machines with a few notable exceptions. Only one HHU device is currently approved for endocavitary use (Clarius EC7 HD); the majority of HHUs are only intended for external scanning or procedural guidance. External scanning over intact skin is considered low risk for infection transmission, so cleaning the probe and cable with a low-level disinfectant is sufficient [26, 27]. US-guided procedures are considered high risk and require a barrier (Tegaderm, probe cover) plus low-level disinfection.

One notable difficulty that has arisen during the SARS$\mathrm{CoV}-2$ pandemic is machine disinfection between suspected or confirmed cases. Given documented persistence of SARS$\mathrm{CoV}-2$ on surfaces up to 3 days, current recommendations are 
to drape cart-based machines with disposable plastic drapes. This process is time-consuming and resource-intensive. HHU devices present a solution for POCUS infection control as there is no cart-based system in existence that is as easily cleaned or covered [11, 16, 28, 29]. HHU devices can be quickly and completely cleaned with a single disinfectant wipe. Another simple alternative is to place the entire US device and tablet/phone within a sterile probe cover and close the end [30]. After the exam is complete, the probe cover is discarded and the device wiped down. Additionally, the remote viewing capabilities offered by HHU technologies may be beneficial for infection control as they allow one user to obtain images that can be virtually viewed by other clinicians outside the patient room [5].

\section{Current Use of HHUs in the ED}

POCUS applications in the ED using traditional cart-style machines are well established as standard of care for the management of certain disease processes [24]. Use of HHU devices in the ED is currently informed by mostly small, singlecenter studies with few randomized control trials (RCTs) available at this time. Nonetheless, the limited data from these studies is promising [3, 8, 31-35]. A retrospective study evaluating POCUS lung scores obtained from ED COVID-19 patients using a Butterfly iQ HHU and traditional GE Venue machine reported a high degree of correlation between images [31]. Two other studies using HHU to differentiate the cause of acute dyspnea in the ED found HHU use feasible for this purpose and demonstrated reliable discrimination between cardiac and non-cardiac causes of dyspnea [33, 34]. Pujol et al. determined that it is feasible to diagnose proximal deep vein thrombosis by performing venous compression studies with a HHU device [32]. Another small RCT found no difference between HHU and traditional machines when used to guide EPs placing IJ central lines on training models [35]. Similarly, a small retrospective study found HHU devices feasible to use for ultrasound-guided paracentesis [36].

Though evidence on HHU use for traditional POCUS applications in the ED is sparse, handhelds already demonstrate great potential to change specific areas of EM practice. One such area is within the scope of ED patient resuscitation. Though no large studies have been performed assessing the non-inferiority of HHU for rapid evaluation ED patients in this context, it stands to reason that smaller, portable equipment would be beneficial in these circumstances. Some data exists that hints HHU would perform adequately as a standalone device if used during resuscitation efforts. Previous comparison studies between HHU and standard machines reported general agreement for evaluation of left ventricular ejection fraction, pericardial effusions, and inferior vena cava collapsibility, three clinical questions frequently evaluated by
EPs attending critically ill patients [37]. Another study found the results of focused assessment with sonography in trauma (FAST) assessments of trauma patients performed by EPs using HHU devices correlated well with findings from radiologist performed abdominal ultrasounds that immediately followed, suggesting that HHU performs as well as standard machines for this application [38].

HHU also exhibits value in areas of patient care previously prohibitive to widespread use of ultrasound due to constraints inherent in traditional machines. In some EDs, trained ancillary staff perform POCUS peripheral IV (PIV) placement. Until recently this has been executed using standard machines, leading to workflow issues when a machine needed for a POCUS study by an EP is in use by another ED team member placing IVs. HHU devices offer a solution to this problem, easily replacing larger ultrasound machines as the workhorses for ultrasound PIV placement, thus freeing the main machines for more complex POCUS studies. A retrospective study of PIVs placed in difficult to access ED patients using the Philips Lumify HHU system found 92\% success with HHU devices, compared to the $97 \%$ success rate using traditional ultrasound machines; first-attempt success was similar as well [39•].

One of the most robust areas of application for HHU devices outside the ED is medical education. HHU, like its larger predecessors, can be utilized in undergraduate and graduate medical education to teach safer approaches to invasive procedures, improve physical examinations, and enhance understanding of anatomy [5]. Some evidence suggests that physicians are less inclined to utilize physical examination skills and that the quality and proficiency of these skills is lacking and prone to error $[40,41]$. POCUS is proven, to improve bedside physical exams by medical students and residents to the point that one author recommends "insonation" as a component of the physical exam [4, 40, 42]. Recent studies demonstrate that incorporating $\mathrm{HHU}$ devices into undergraduate medical education was well received by students, improved conceptual understanding of anatomy, and improved student confidence and diagnostic accuracy when performing physical examinations [42-44, 45••, 46].

POCUS use is widespread in the clinical setting, utilized by practitioners spanning multiple specialties. In order for learners to keep up-to-date with the myriad clinical ultrasound applications, medical schools are integrating concepts of ultrasonography — such as teaching bedside image acquisition and image interpretation - into their core medical school curricula $[44,47]$. In a survey of ultrasound medical education directors, respondents noted that lack of funding was a huge barrier to implementing ultrasound curricula for medical students [44]. With the price of some HHU devices around $\$ 2000$ (compared to $\$ 50,000$ or greater for a cart-based system), they represent a low-cost solution to providing the benefits of early ultrasound education to medical students. The feasibility of this is demonstrated by the medical school at the 
University of California Irvine which provided Butterfly iQ devices to their entire 2023 medical student class [6].

HHU has great potential for use outside of traditional hospital and academic settings $[3,48]$. The use of POCUS to augment the work of paramedics and first responders has been previously explored, but the size, cost, and necessity for multiple probes inherent to standard machines significantly limited the ability to undertake large scale prehospital studies [48]. With the advent of HHU devices, research on prehospital POCUS and its effect on patient outcomes is now feasible [49]. The cost and portability of HHU devices make them ideal diagnostic tools for first responders as well as EPs who staff large-scale events, sometimes in areas with few existing medical resources [4]. Large gatherings can generate disease states warranting POCUS imaging such as trauma, dehydration, and undifferentiated dyspnea [50]. Prager et al. prospectively evaluated the use of HHU by EPs at a large music festival and reported that HHU findings altered the management decision in $39 \%$ of cases. Prager notes that, "As POCUS machines continue to become more portable and less expensive, this modality could prove to be an important measure to reduce downstream health care costs in patients presenting [for evaluation] at mass gatherings" [50]. There are also documented cases of HHU assisting in triage during disaster/ mass-casualty scenarios [48]. Their high portability and ability to provide real-time diagnostics make these devices ideal for such circumstances [4]. Similarly, HHU is proving invaluable to practitioners in austere environments that otherwise have no reliable imaging source [3, 4, 15, 49]. Burleson et al. retrospectively reported the experience of ultrasound-trained physicians using the Butterfly iQ HHU in an austere East African ED [8]. They found that while there were some disadvantages to the device, such as frequent overheating and poorer quality of cardiac POCUS, the Butterfly iQ met the needs of their high-acuity ED [8]. The authors note that "...advantages over cart-based machines are magnified where financial resources, floor space, and reliable power may be scarce" [8].

While evidence for the use of HHU within the scope of EM practice is in its infancy, more rigorous data on the subject exists in literature outside of this specialty [3, 4, 32, 37, 51, 52]. Findings from some of these studies are undeniably applicable to HHU use in the ED. A prospective blinded study comparing HHU devices to traditional machines for grading hydronephrosis found excellent agreement between the two devices [51]. A systematic review comparing HHU devices to standard machines reported that "Strong correlations [between devices] were found when evaluating ascites, hydronephrosis, pleural cavities, in detection of abdominal aortic aneurysms and for use with obstetric and gynecological patients" [3••]. Overall, there remains a paucity of data generated from large RCTs directly comparing handheld devices to cart-style machines in the context of ED use. Data from such studies are necessary before broad adoption of HHU as the primary POCUS tool in the ED is possible, but the potential of these devices is already apparent.

\section{Future of Handhelds in the ED}

Remotely guided US, or "tele-ultrasound," improves image quality and scanning confidence among novice users and will be critical for improving safety as HHU becomes more widespread [5, 15, 53, 54]. AI algorithms have been developed that automatically show users the movements necessary to obtain high quality images $[5,55]$. Use of neural networks in computer science also has significant implications for POCUS [56]. Many HHU devices already possess AI functions such as automated calculation of ejection fraction and bladder volume $[57,58]$. Zhang et al. created a fully automated echocardiography interpretation algorithm which functions with Bmode images obtained by HHU [59]. Thus, practitioners using machines lacking advanced echocardiography functionality or high-end image quality may still use HHU devices to answer cardiac-related clinical questions due to these advances in $\mathrm{AI}$ and smart device processing power. The combination of widespread HHU availability, evolving tele-ultrasound capabilities and machine learning algorithms paints a promising picture for patient care with earlier, more accurate disease detection from even novice users, in any geographic location [3••].

Concordant with advances in tele-ultrasound, virtual POCUS education has recently become feasible. Technologies inherent in some HHU devices allow for remote image viewing and thus remote peer-review and QA [5]. HHU devices saw increased use as adjuncts to resident education during the COVID-19 pandemic due to their compatibility with virtual platforms [60]. Research on efficacy of this emerging facet of medical education is currently extremely limited but likely to expand in the face of restrictions on inperson learning during the current COVID-19 pandemic.

HHU will likely see increased future use by ED consultants, including specialties that are not traditional users of POCUS (e.g., nephrology, internal medicine) [61]. The likelihood that HHU devices will become ubiquitous outside the ED relatively soon has led some to suggest that US should become a routine part of the physical examination $[3 \bullet \bullet, 40$, 62]. As US education is increasingly incorporated into medical school curricula, it is likely to make specialty-specific training and implementation at the graduate level easier and more widespread [5, 63-65].

Use of HHU devices by consultants offers many potential benefits for patient care. In an office-based setting, it could be used to identify conditions requiring urgent ED referral (e.g., large pericardial effusion) versus those appropriately treated in the outpatient setting (e.g., cellulitis without abscess). In the inpatient setting, HHU could be used to track serial changes in a patient's condition, such as pulmonary edema, from arrival in the ED until discharge from the floor. Alternatively, consultants could use HHU devices to perform focused examinations to answer limited questions - has left ventricular ejection fraction decreased or does the patient with known 
cholelithiasis now have findings suggestive of acute cholecystitis? Rapid answers to these direct questions, particularly with consultants at the bedside, could facilitate treatment and disposition decisions while reducing the burden on sonographers and interpreting physicians [ $3 \bullet \cdot]$.

While it is highly plausible that HHU use can improve patient care, demonstration of such benefit with formal research studies will strengthen the argument for widespread implementation and direct its use to the areas with greatest potential impact. Several preliminary studies suggest benefits of HHU use. These include reduced need for follow-up testing when used in the outpatient setting, with a low incidence of false negatives (5\%), and faster time to diagnosis and reduced time to first intervention when HHUs were used by rapid response teams $[66,67]$. While image interpretability appears similar between HHUs and cart-based systems, a critical concept for future studies is not to conflate image quality and the setting of use with operator training and skills [68]. Maintenance of rigorous educational programs and adequate quality assurance are crucial as device availability increases.

\section{Conclusion}

The potential benefits of a handheld advanced imaging system are undeniable. With further technologic developments, the gap in functionality between handheld and cart-based systems will continue to decline.

Though there are obstacles surrounding HHU device operation and image management, many of these can be overcome through administrative hospital support and the fiscal advantages these devices provide. Medical education continues to be a driving force for HHU innovation and adoption. Training a new generation of physicians in anatomy and diagnostics using these platforms will foster familiarity and comfort that is likely continue into regular practice.

As we look toward the future of ultrasound, the growing body of research to improve and validate use of handheld ultrasound devices will be key to improving patient outcomes and expanding its use to novel settings. Implementation of machine learning and tele-ultrasound applications is likely to alter the POCUS landscape, and physicians should continue to study and embrace these technologies for the improvement of patient care.

\section{Declarations}

Conflict of Interest The authors declare no competing interests.

Human and Animal Rights and Informed Consent This article does not contain any studies with human or animal subjects performed by any of the authors.

\section{References}

Papers of particular interest, published recently, have been highlighted as:

- Of importance

- Of major importance

1. American College of Emergency Physicians. Appropriate use criteria for handheld pocket ultrasound devices: ACEP Policy Statement. https://www.acep.org/globalassets/new-pdfs/policystatements/appropriate-use-criteria-for-handheld-pocketultrasound-devices.pdf. Published July 2018. Approved June 2018. Accessed December 2020. ACEP Statement describing handheld ultrasound images as "comparable" to cart-based machines.

2. Liu R. What's the deal with pocket ultrasound?. ACEP Now, [online] 2020;38(7). Available at: <https://www.acepnow.com/article/ whats-the-deal-with-pocket-ultrasound/>. Accessed 12 December 2020.

3.• Rykkje A, Carlsen JF, Nielsen MB. Hand-held ultrasound devices compared with high-end ultrasound systems: a systematic review. Diagnostics (Basel). 2019;9(2):61. https://doi.org/10.3390/ diagnostics 9020061 . Systematic review of $\mathbf{1 6}$ publications comparing HHU to cart-based machines for abdominal and lung applications. The review found good agreement between device types for several specific examinations but noted that research is needed.

4. European Society of Radiology (ESR). ESR statement on portable ultrasound devices. Insights Imaging. 2019 Sep 16;10(1):89. https://doi.org/10.1186/s13244-019-0775-x.

5. Baribeau Y, Sharkey A, Chaudhary O, Krumm S, Fatima H, Mahmood F, et al. Handheld point-of-care ultrasound probes: the new generation of POCUS. J Cardiothorac Vasc Anesth. 2020 Nov;34(11):3139-45. https://doi.org/10.1053/j.jvca.2020.07.004.

6. Butterfly Network. Tonight, @UCIrvine introduced the medical professionals of the future! Every incoming UC Irvine med school student was presented with their very own \#ButterflyiQ. https://t.co/ TzrJvxU21h [Internet]. @ButterflyNetInc. 2019 [cited 2021 Feb 20]. Available from: https://www.twitter.com/ButterflyNetInc/ status/1160024249211314176.

7. Kodaira Y, Pisani L, Boyle S, Olumide S, Orsi M, Adeniji AO, et al. Reliability of ultrasound findings acquired with handheld apparatuses to inform urgent obstetric diagnosis in a high-volume resource-limited setting. Int J Gynecol Obstet. 2020. https://doi.org/ 10.1002/ijgo.13475.

8. Burleson SL, Swanson JF, Shufflebarger EF, Wallace DW, Heimann MA, Crosby JC, et al. Evaluation of a novel handheld point-of-care ultrasound device in an African emergency department. Ultrasound J. 2020 Dec 7;12(1):53. https://doi.org/10.1186/ s13089-020-00200-8.

9. Zardi EM, Franceschetti E, Giorgi C, Palumbo A, Franceschi F. Accuracy and performance of a new handheld ultrasound machine with wireless system. Sci Rep. 2019 Oct 10;9(1):14599. https://doi. org/10.1038/s41598-019-51160-6.

10.• Galusko V, Bodger O, Ionescu A. A systematic review of pocketsized imaging devices: small and mighty? Echo Res Pract. 2018 Dec:113-38. A systematic review of HHU use for cardiac applications by users with various levels of ultrasound training. Found HHU was feasibly used by all training groups though with some noted decreased diagnostic accuracy. States that HHU may serve well as a screening exam for pathology prior to formal echo studies. Noted a need for further studies in this area due to the heterogeneity of those currently available. 
11. Thavanathan RS, Woo MY, Hall G. The future is in your hands Handheld ultrasound in the emergency department. CJEM. 2020 Aug;12:1-3. https://doi.org/10.1017/cem.2020.449.

12. Mancusi C, Carlino MV, Sforza A. Point-of-care ultrasound with pocket-size devices in emergency department. Echocardiography. 2019;36(9):1755-64. https://doi.org/10.1111/echo.14451. Review article describing the use of handheld POCUS in the ED and its feasibility for use in acute dyspnea, shock and chest pain patients.

13. Butterfly Network. Tonight, @UCIrvine introduced the medical professionals of the future! Every incoming UC Irvine med school student was presented with their very own \#ButterflyiQ. https://t.co/ TzrJvxU21h [Internet]. @ButterflyNetInc. 2019 [cited 2021 Feb 20]. Available from: https://twitter.com/ButterflyNetInc/ status/1160024249211314176.

14. Wilkinson J, Saxhaug L. Handheld ultrasound in training - The future is getting smaller!. J Intensive Care Soc, [online]; 2020;175114372091421. Available at: https://journals.sagepub. com/doi/full/10.1177/1751143720914216. Accessed 12 December 2020.

15. Salerno A, Tupchong K, Verceles AC, McCurdy MT. Point-of-care Teleultrasound: a systematic review. Telemed J E Health. 2020 Nov;26(11):1314-21. https://doi.org/10.1089/tmj.2019.0177.

16. TeleGuidance - Butterfly Network [Internet]. [cited 2021 Feb 20]. Available from: https://www.butterflynetwork.com/teleguidance.

17. Shini SG, Thomas T, Chithraranjan K. Cloud based medical image exchange-security challenges. Procedia Eng. 2012;38:3454-61.

18. Butterfly IQ Patient Privacy Notice. https://www.butterflynetwork. com/patient-privacy. Accessed February 14, 2021.

19. Trice Data Protection Policy. https:/triceimaging.com/data/. Accessed February 14, 2021.

20. Butterfly Network Global Privacy. FAQs https://www. butterflynetwork.com/global-privacy. Accessed February 14, 2021.

21. Stites M, Pianykh O. How secure is your radiology department? mapping digital radiology adoption and security worldwide. Am J Rad. 2016;206:797-804.

22. "Millions of Americans' medical images and data are available on the internet. Anyone can take a peek." https://www.propublica.org/ article/millions-of-americans-medical-images-and-data-areavailable-on-the-internet

23. Can cloud-based archiving cure your security headaches?: Your medical imaging cloud. Ambra Health. https://ambrahealth.com/ hospitals-health-systems/can-cloud-based-archiving-cure-securityheadaches/. Published August 22, 2017. Accessed February 14, 2021.

24. American College of Emergency Physicians. ACEP Ultrasound Guidelines: Emergency, point-of-care and clinical ultrasound guidelines in medicine: ACEP Policy Statement. https://www. acep.org/patient-care/policy-statements/ultrasound-guidelinesemergency-point-of\%2D\%2Dcare-and-clinical-ultrasoundguidelines-in-medicine/. Published October 2008, revised June 2016. Accessed January 2021

25. Geria RN, Raio CC, Tayal V. Point-of-care ultrasound: not a stethoscope-a separate clinical entity. J Ultrasound Med. 2015;34:172-3.

26. Reprocessing Requirements for Ultrasound Probes (College of Physicians and Surgeons of British Columbia). https://www. cpsbc.ca/files/pdf/Reprocessing-Requirements-Ultrasound-Probes. pdf.

27. American College of Emergency Physicians. ACEP guideline for ultrasound transducer cleaning and disinfection. https://www.acep. org/patient-care/policy-statements/guideline-for-ultrasoundtransducer-cleaning-and-disinfection/. Published June 2018. Accessed January 2021.

28. Kirkpatrick AW, McKee JL, Volpicelli G, Ma IWY. The potential for remotely mentored patient-performed home self-monitoring for new onset alveolar-interstitial lung disease. Telemed J E Health. 2020 Oct;26(10):1304-7. https://doi.org/10.1089/tmj.2020.0078.

29. Khanji MY, Ricci F, Patel RS, Chahal AA, Bhattacharyya S, Galusko V, et al. Special Article - The role of hand-held ultrasound for cardiopulmonary assessment during a pandemic. Prog Cardiovasc Dis. 2020 Sep-Oct;63(5):690-5. https://doi.org/10. 1016/j.pcad.2020.07.003.

30. Gibson LE, Bittner EA, Chang MG. Handheld ultrasound devices: An emerging technology to reduce viral spread during the Covid-19 pandemic. Am J Infect Control. 2020;48(8):968-9. https://doi.org/ 10.1016/j.ajic.2020.05.041

31. Bennett D, De Vita E, Mezzasalma F, Lanzarone N, Cameli P, Bianchi F, et al. Portable pocket-sized ultrasound scanner for the evaluation of lung involvement in coronavirus disease 2019 patients. Ultrasound Med Biol. 2021 Jan;47(1):19-24. https://doi. org/10.1016/j.ultrasmedbio.2020.09.014.

32. Pujol S, Laurent J, Markarian T, Claret PG, Lefrant JY, Roger C, et al. Compression with a pocket-sized ultrasound device to diagnose proximal deep vein thrombosis. Am J Emerg Med. 2018 Jul;36(7): 1262-4. https://doi.org/10.1016/j.ajem.2018.03.076.

33. Kirkpatrick AW, McKee I, McKee JL, Ma I, McBeth PB, Roberts DJ, et al. Remote just-in-time telementored trauma ultrasound: a double-factorial randomized controlled trial examining fluid detection and remote knobology control through an ultrasound graphic user interface display. Am J Surg. 2016 May;211(5):894-902.e1. https://doi.org/10.1016/j.amjsurg.2016.01.018.

34. Sforza A, Mancusi C, Carlino MV, Buonauro A, Barozzi M, Romano G, et al. Diagnostic performance of multi-organ ultrasound with pocket-sized device in the management of acute dyspnea. Cardiovasc Ultrasound. 2017 Jun 19;15(1):16. https://doi. org/10.1186/s12947-017-0105-8.

35. Chetioui A, Masia T, Claret PG, Markarian T, Muller L, Lefrant JY, et al. Pocket-sized ultrasound device for internal jugular puncture: A randomized study of performance on a simulation model. J Vasc Access. 2019 Jul;20(4):404-8. https://doi.org/10.1177/ 1129729818812733.

36. Keil-Ríos D, Terrazas-Solís H, González-Garay A, Sánchez-Ávila JF, García-Juárez I. Pocket ultrasound device as a complement to physical examination for ascites evaluation and guided paracentesis. Intern Emerg Med. 2016 Apr;11(3):461-6. https:// doi.org/10.1007/s11739-016-1406-X.

37. Platz E, Pivetta E, Merz AA, Peck J, Rivero J, Cheng S. Impact of device selection and clip duration on lung ultrasound assessment in patients with heart failure. Am J Emerg Med. 2015;33(11):1552-6. https://doi.org/10.1016/j.ajem.2015.06.002.

38. Coșkun F, Akıncı E, Ceyhan MA, Sahin KH. Our new stethoscope in the emergency department: handheld ultrasound. Ulus Travma Acil Cerrahi Derg. 2011 Nov;17(6):488-92. https://doi.org/10. 5505/tjtes.2011.89914.

39. Acuña J, Sorenson J, Gades A, Wyatt R, Stea N, Drachman M, et al. Handheld ultrasound: overcoming the challenge of difficult peripheral intravenous access in the emergency department. J Ultrasound Med. 2020;39(10):1985-91. https://doi.org/10.1002/jum.15303.

Retrospective review that found a specific HHU device reliable for PIV placement in patients with difficult IV access.

40. Narula J, Chandrashekhar Y, Braunwald E. Time to add a fifth pillar to bedside physical examination: inspection, palpation, percussion, auscultation, and insonation. JAMA Cardiol. 2018 Apr 1;3(4):346-50. https://doi.org/10.1001/jamacardio.2018. 0001.

41. Oliver CM, Hunter SA, Ikeda T, Galletly DC. Junior doctor skill in the art of physical examination: a retrospective study of the medical admission note over four decades. BMJ Open. 2013;3(4):e002257.

42. Wong CK, Hai J, Chan KYE, et al. Point-of-care ultrasound augments physical examination learning by undergraduate medical students. Postgrad Med J. 2021;97:10-5. 
43. Ireson M, Warring S, Medina-Inojosa JR, O'Malley MT, Pawlina W, Lachman N, et al. First year medical students, personal handheld ultrasound devices, and introduction of insonation in medical education. Ann Glob Health. 2019 Oct 15;85(1):123. https://doi. org/10.5334/aogh.2565.

44. Dinh VA, Fu JY, Lu S, Chiem A, Fox JC, Blaivas M. Integration of Ultrasound in Medical Education at United States Medical Schools. J Ultrasound Med. 2016;35:413-9. https://doi.org/10.7863/ultra. 15.05073 .

45.• Tarique U, Tang B, Singh M, Kulasegaram KM, Ailon J. Ultrasound curricula in undergraduate medical education: a scoping review. J Ultrasound Med. 2018;37:69-82 https://doi-org. proxy.kumc.edu/10.1002/jum.14333. An review article on the benefits of ultrasound integration into undergraduate medical education noting that POCUS in the curriculum improves medical student physical examination and procedural skills as well as improves clinical knowledge. Specifically notes that handheld ultrasound improved detection of rheumatic heart disease by trainees when compared to history and physical exam alone.

46. Cawthorn TR, Nickel C, O'Reilly M, Kafka H, Tam JW, Jackson LC, et al. Development and evaluation of methodologies for teaching focused cardiac ultrasound skills to medical students. J Am Soc Echocardiogr. 2014 Mar;27(3):302-9. https://doi.org/10.1016/j. echo.2013.12.006

47. Bahner DP, Goldman E, Way D, Royall NA, Liu YT. The state of ultrasound education in U.S. Medical Schools. Acad Med. December 2014;89(12):1681-6. https://doi.org/10.1097/ACM. 0000000000000414

48. Ma OJ, Norvell JG, Subramanian S. Ultrasound applications in mass casualties and extreme environments. Crit Care Med. 2007 May;35(5 Suppl):S275-9.

49. Amaral CB, Ralston DC, Becker TK. Prehospital point-of-care ultrasound: A transformative technology. SAGE Open Med. $2020 \mathrm{Jul}$ 26;8:2050312120932706. https://doi.org/10.1177/ 2050312120932706.

50. Prager R, Sedgwick C, Lund A, Kim D, Ho B, Stachura M, et al. Prospective evaluation of point-of-care ultrasound at a remote, multi-day music festival. Prehosp Disaster Med. 2018 Oct;33(5): 484-9. https://doi.org/10.1017/S1049023X18000821.

51. Kameda T, Uebayashi K, Wagai K, Kawai F, Taniguchi N. Assessment of the renal collecting system using a pocket-sized ultrasound device. J Med Ultrason (2001). 2018 Oct;45(4):57781. https://doi.org/10.1007/s10396-018-0881-2.

52. Mantella LE, Colledanchise K, Bullen M, Hétu MF, Day AG, McLellan CS, et al. Handheld versus conventional vascular ultrasound for assessing carotid artery plaque. Int J Cardiol. 2019 Mar 1;278:295-9. https://doi.org/10.1016/j.ijcard.2018.12.014.

53. Kirkpatrick AW, McKee I, McKee JL, Ma I, McBeth PB, Roberts DJ, et al. Remote just-in-time telementored trauma ultrasound: a double-factorial randomized controlled trial examining fluid detection and remote knobology control through an ultrasound graphic user interface display. Am J Surg. 2016 May;211(5):894-902.e1. https://doi.org/10.1016/j.amjsurg.2016.01.018.

54. Jensen SH, Weile J, Aagaard R, Hansen KM, Jensen TB, Petersen $\mathrm{MC}$, et al. Remote real-time supervision via tele-ultrasound in focused cardiac ultrasound: A single-blinded cluster randomized controlled trial. Acta Anaesthesiol Scand. 2019 Mar;63(3):403-9. https://doi.org/10.1111/aas.13276.

55. Commissioner $\mathrm{O}$ of the FDA Authorizes Marketing of First Cardiac Ultrasound Software That Uses Artificial Intelligence to Guide User [Internet]. FDA. FDA; 2020 [cited 2021 Feb 20]. Available from: https://www.fda.gov/news-events/press-announcements/fdaauthorizes-marketing-first-cardiac-ultrasound-software-usesartificial-intelligence-guide-user.

56. Shokoohi H, LeSaux MA, Roohani YH, Liteplo A, Huang C, Blaivas M. Enhanced point-of-care ultrasound applications by integrating automated feature-learning systems using deep learning. J Ultrasound Med. 2019 Jul;38(7):1887-97.

57. The Latest in Cardiovascular Hand-Held Point-of-Care Ultrasound: The Power of Echocardiography Anytime, Anywhere - American College of Cardiology [Internet]. [cited $2021 \mathrm{Feb} 20$ ]. Available from: https://www.acc.org/latest-in-cardiology/articles/2019/08/ 12/08/11/the-latest-in-cardiovascular-hand-held-point-of-careultrasound.

58. Automatically Estimate Bladder Volume [Internet]. Butterfly Network. [cited $2021 \mathrm{Feb} 20]$. Available from: https://support. butterflynetwork.com/hc/en-us/articles/360042212671Automatically-Estimate-Bladder-Volume

59. Jeffrey Z, Sravani G, Pulkit A, Tison GH, Hallock LA, Lauren B-N, et al. Fully automated echocardiogram interpretation in clinical practice. Circulation. 2018 Oct 16;138(16):1623-35.

60. Lin SD. A virtual point-of-care ultrasound course during the COVID-19 pandemic. AEM Educ Train. 2020 Nov 9. https://doi. org/10.1002/aet2.10545.

61. Soomro QH, Amerling R. Point-of-care ultrasound in nephrology. Curr Opin Nephrol Hypertens. 2021 Mar 1;30(2):176-83. https:// doi.org/10.1097/MNH.0000000000000681.

62. Koratala A. Focus on POCUS: it is time for the kidney doctors to upgrade their physical examination. Clin Exp Nephrol. 2019 Jul;23(7):982-4. https://doi.org/10.1007/s10157-019-01707-8.

63. Liu RB, Suwondo DN, Donroe JH, Encandela JA, Weisenthal KS, Moore CL. Point-of-care ultrasound: does it affect scores on standardized assessment tests used within the preclinical curriculum? J Ultrasound Med. 2019 Feb;38(2):433-40. https://doi.org/10.1002/ jum. 14709 .

64. Johri AM, Durbin J, Newbigging J, Tanzola R, Chow R, De S, et al. Cardiac point-of-care ultrasound: state-of-the-art in medical school education. J Am Soc Echocardiogr. 2018 Jul;31(7):749-60. https:// doi.org/10.1016/j.echo.2018.01.014.

65. Ma IWY, Steinmetz P, Weerdenburg K, Woo MY, Olszynski P, Heslop CL, et al. The canadian medical student ultrasound curriculum: a statement from the canadian ultrasound consensus for undergraduate medical education group. J Ultrasound Med. 2020 Jul;39(7):1279-87. https://doi.org/10.1002/jum.15218.

66. Colli A, Prati D, Fraquelli M, Segato S, Vescovi PP, Colombo F, et al. The use of a pocket-sized ultrasound device improves physical examination: results of an in- and outpatient cohort study. PLoS One. 2015 Mar 20;10(3):e0122181. https://doi.org/10.1371/ journal.pone.0122181.

67. Zieleskiewicz L, Lopez A, Hraiech S, Baumstarck K, Pastene B, Di Bisceglie M, et al. Bedside POCUS during ward emergencies is associated with improved diagnosis and outcome: an observational, prospective, controlled study. Crit Care. 2021 Jan 22;25(1):34. https://doi.org/10.1186/s13054-021-03466-z.

68. Dewar ZE, Wu J, Hughes H, Adnani A, Christiansen G, Ovedovitz $\mathrm{L}$, et al. A comparison of handheld ultrasound versus traditional ultrasound for acquisition of RUSH views in healthy volunteers. $\mathrm{J}$ Am Coll Emerg Physicians Open. 2020 Nov 21;1(6):1320-5. https://doi.org/10.1002/emp2.12322.

Publisher's Note Springer Nature remains neutral with regard to jurisdictional claims in published maps and institutional affiliations. 